\title{
Fortschritte der Röntgen- und Radiumstrahlen-Forschung
}

Ein neues Rönígenaufnahmeveríahren. Von Ing. Friedrich Dessauer und Dr. B. Wiesner in Aschaffenburg. Münchner med. Wochenschr.

1909. No. 44. Die bisher mit den neuen Intensivstrom-Induktoren erreielite Herab-setzung der Expositionszeit auf eine Sekunde gestattete noch nicht, scharfe Bilder von in Bewegung befindlichen Organen zu erhalten. Dessauer gelang es, einen Apparat zu konstruieren, der eine weitere Kürzung der Exposition auf 1/50-1/100 Sekunde ermöghchte. Bei Verzicht auf den bisher üblichen Unterbrecher wird in den primären Stromkreis eine Patrone eingeschaltet, die bei einer gewissen Strom-stärke platzt und damit den Strom unterbricht. Hierdurch wird der Sekundärspule ein sehr hräftiger Stromstoss induziert und die Röhren zum Aufblitzen gebracht. Die Wucht der Induktion wird durch die Stärke des in den Patronen befindlichen Schmelzdrahtes bestimmt; schwächere Drähte für Extremitätenaufnahmen, stärkere für Thorax, Kopf und Becken.

Literatur-Verzeichnis.

561

Experimentelle üntersuchungen über die Einwirkung der Röntgen-strahlen auf die Linse. Von Dr. Bosstiet Alphonse in Goa (Ostindien). Arch. f. Augenheilk. Bd. LXIV. Heft 3.

Die Untersuchungen wurden mit einem Röntgen-Apparat von $50 \mathrm{~cm}$ Funkenlänge bei 10 bis 12 Ampere Stromstärke und 3-10 cm Röhren-abstand ausgeführt; die Belichtungsdauer betrug 3-90 Minuten. Zur Belichtung kamen Linsen noch ungeborener, neugeborener und er-wachsener ïiere. Bei alien 3 Gruppen liessen sich Schädigungen der Linse feststellen, im wesentlichen gekennzeichnet durch Degeneration des Kapselepithels und der Rindensubstanz, iiauptsächlich am Áquator und in der hinteren Corticalis. Daneben fand sich beginnende Regeneration des Linsenepithels, die zur Bildung grosser Zellkomplexe - Epithelnester - führte. Es zeigte sich, dass die schädliche Wirkung der Strahlen um so mehr hervortrat, je jünger das Gewebe war. Dr. E1/8ers $7 / 8$ eirø-Bonn.

Literatur - Verzeichnis.

Anatomie. - Physiologie. - Pathologie.

Adam, C, Beiträge zur Diagnose und Therapie der Augenverletzungen.

Ther. d. Gegenw. 50. Jahrg. 11. H. Armaignac, H., Mensuration et notation du champ visuel. Arch. d'Ophtalm.

Oktober. Ballantgne, Artheiv J'., Transient convulsions in two children with unusual changes in the fundus oculi. Ophthalmoscope. Oktober. Bull, CharlesStedmann, The adverse influence of diabetes in certain operations on the eye. Med. Record. Vol. 76. No. 14. Charles, J. W., The determination of damages from the ocular examination, after an injury. Amer. Journ. of Ophthalm. Oktober. Coats, George, On the occurrence of misplaced derivatives of the secondary optic vesicle in congenitally abnormal eyes. Ophthalmoscope.. Nov. Coullaud, L'appreciation des distances dans la vision binoculaire normale. 
Arch. d'Ophtalm. Oktober. Eberbach, Otto, Weiterer Beitrag zur Kasuistik und Prognose intraokulärer

Tumoren. Diss. Tubingen. Edridge-Green, F. W., The theory of vision. Lancet. No. 4492.

Filipow, N. A., Zur Frage über die Entstehung der Glashaut in der Vorder-

kammer. Klin. Monatsbl. f. Augenheilk. Oktober. Hermann, Th., Zur Myopiefrage und über die

Brillenkorrektion der Kurz-

sichtigen. St. Petersb. med. Wochenschr. No. 43. Heine, Rosa, Ein Beitrag über die sogenannten Vergleichungen über-

merklicher Empfindungsunterschiede. Zeitschr. f. Psych. 54. Bd.

1. u. 2. H. fiillemanns, M., Ueber Sehprüfung bei künstlicher Beleuchtung und Licht-

sinnstörung, besonders bei Myopie. Klin. Monatsbl. f. Augenheilk.

Oktober. Horstmann, C, Die Pathologie und Therapie der sympathischen Ophthalmie.

Deutsche med. Wochenschr. No. 44. Jackson, Edward, Blood pressure in its practical relations to ophthalmology.

Ophthalm.R. Record. Oktober. iM,ndolt,H.

Myopie und Lichtsinn. Klin. Monatsbl. f. Augenheilk. Oktober. Lenz

67.

Zur Pathologie der cerebralen Sehbahn unter besonderer Berück- 